\title{
Exhaled breath hydrogen cyanide as a marker of early Pseudomonas aeruginosa infection in children with cystic fibrosis
}

\author{
Francis J. Gilchrist ${ }^{1,2}$, John Belcher ${ }^{3}$, Andrew M. Jones ${ }^{4}$, David Smith ${ }^{2}$, \\ Alan R. Smyth ${ }^{5}$, Kevin W. Southern ${ }^{6}$, Patrik Španěl ${ }^{7}, A$. Kevin Webb ${ }^{4}$ and \\ Warren Lenney ${ }^{1,2}$ on behalf of the SPACE Study Team ${ }^{8}$
}

Affiliations: ${ }^{1}$ Academic Department of Child Health, University Hospital of North Staffordshire, Stoke on Trent, UK. ${ }^{2}$ Institute of Science and Technology in Medicine, Keele University, Keele, UK. ${ }^{3}$ School of Computing and Mathematics, Keele University, Keele, UK. "Manchester Adult Cystic Fibrosis Centre, University Hospital of South Manchester, Manchester, UK. ${ }^{5}$ Division of Child Health, Obstetrics and Gynaecology, School of Medicine, University of Nottingham, UK. ' Institute of Child Health, Alder Hey Children's Hospital, Liverpool, UK. ' J. Heyrovský Institute of Physical Chemistry, Academy of Sciences of the Czech Republic, Prague, Czech Republic. ${ }^{8}$ For a list of the SPACE Study Team collaborators and their affiliations, see the Acknowledgments section.

Correspondence: Francis J. Gilchrist, Academic Department of Child Health, University Hospital of North Staffordshire, Stoke on Trent, ST4 6QG, UK. E-mail: francis.gilchristवuhns.nhs.uk

ABSTRACT Hydrogen cyanide is readily detected in the headspace above Pseudomonas aeruginosa cultures and in the breath of cystic fibrosis (CF) patients with chronic (P. aeruginosa) infection. We investigated if exhaled breath $\mathrm{HCN}$ is an early marker of $P$. aeruginosa infection.

233 children with CF who were free from $P$. aeruginosa infection were followed for 2 years. Their median (interquartile range) age was 8.0 (5.0-12.2) years. At each study visit, an exhaled breath sample was collected for hydrogen cyanide analysis. In total, 2055 breath samples were analysed. At the end of the study, the hydrogen cyanide concentrations were compared to the results of routine microbiology surveillance.

$P$. aeruginosa was isolated from 71 children during the study with an incidence (95\% CI) of 0.19 (0.15-0.23) cases per patient-year. Using a random-effects logistic model, the estimated odds ratio ( $95 \%$ CI) was 3.1 (2.6-3.6), which showed that for a 1-ppbv increase in exhaled breath hydrogen cyanide, we expected a $212 \%$ increase in the odds of $P$. aeruginosa infection. The sensitivity and specificity were estimated at $33 \%$ and $99 \%$, respectively.

Exhaled breath hydrogen cyanide is a specific biomarker of new $P$. aeruginosa infection in children with CF. Its low sensitivity means that at present, hydrogen cyanide cannot be used as a screening test for this infection.

@ERSpublications

Exhaled hydrogen cyanide is a specific but insensitive biomarker of new $P$. aeruginosa infection in children with CF http://ow.ly/TdRaF

Support statement: We gratefully acknowledge the funding for the SPACE Study from a Research for Patient Benefit Grant from the UK National Institute of Health Research (NIHR) (grant PB-PG-0909-20070). This report presents independent research funded by the NIHR. The views expressed are those of the authors and not necessarily those of the UK National Health Service, the NIHR or the UK Department of Health. Funding information for this article has been deposited with FundRef.

Conflict of interest: None declared.

The content of this work is (C)the authors or their employers. Design and branding are (CERS 2015. This article is open access and distributed under the terms of the Creative Commons Attribution Non-Commercial Licence 4.0 


\section{Introduction}

Chronic Pseudomonas aeruginosa infection has a detrimental effect on morbidity and mortality in patients with cystic fibrosis (CF) [1,2]. This can be prevented by early detection and treatment [3, 4]. As a significant proportion of patients with new $P$. aeruginosa infection remain asymptomatic [5], microbiological surveillance is vital to ensure that it is not missed. Unfortunately, this is difficult in children as they are often unable or unwilling to expectorate sputum. In such patients, $P$. aeruginosa detection is dependent on cough swabs, which are unreliable [6,7]; on sputum induction, which is time consuming and expensive $[8,9]$; or on bronchoalveolar lavage (BAL), which is invasive and requires a general anaesthetic [10]. The urgent need for a child-friendly, noninvasive method to accurately detect $P$. aeruginosa infection has led to interest in developing a diagnostic exhaled breath test.

The most extensively investigated biomarker of $P$. aeruginosa that could be used in an exhaled breath test is hydrogen cyanide. It has been known for more than a century that $P$. aeruginosa produces nonvolatile cyanide ions [11]. More recently, selected ion flow tube mass spectroscopy (SIFT-MS) has been used to detect and analyse volatile hydrogen cyanide released into the gas phase by cultures of $P$. aeruginosa [12]. The in vitro conditions affecting hydrogen cyanide release have been established $[13,14]$ and it is known that $P$. aeruginosa is the only organism frequently found in the CF lung that produces hydrogen cyanide $[15,16]$. In vivo studies have demonstrated elevated exhaled breath hydrogen cyanide in both children and adults with CF and chronic P. aeruginosa infection, compared to controls [17, 18]. These studies demonstrated that hydrogen cyanide is a biomarker of chronic P. aeruginosa infection. As designed, they could not reveal if hydrogen cyanide was an early marker of $P$. aeruginosa infection, which is necessary if it is to be a used as a screening tool. The SPACE (Sensitivity and Specificity of Pseudomonas aeruginosa Detection Using the Hydrogen Cyanide Concentration of Exhaled Breath) Study was designed to investigate this. The aims of the SPACE Study were 1) to investigate if breath hydrogen cyanide is a biomarker of early $P$. aeruginosa infection in children with CF and 2) to collect prospective data on the incidence rate of new $P$. aeruginosa infection in children with CF who were previously free from $P$. aeruginosa infection

\section{Methodology}

To achieve the study's aims, exhaled breath hydrogen cyanide concentration had to be measured in a group of children with $\mathrm{CF}$ around the time of a new $P$. aeruginosa infection. As the onset of infection cannot be predicted, a large cohort of children who were free from $P$. aeruginosa infection was recruited on the presumption that a proportion would acquire $P$. aeruginosa during the study. At each CF outpatient appointment, in addition to the respiratory microbiology sample taken as part of routine care, an exhaled breath sample was collected for hydrogen cyanide analysis and a clinical details questionnaire completed. At the end of the study, the microbiology results were compared to the breath hydrogen cyanide concentrations and the clinical questionnaire results.

\section{Inclusion criteria}

The inclusion criteria were: 1 ) children 2-16 years old with CF; 2) no P. aeruginosa isolated from the child in the preceding 12 months; 3 ) all routine CF appointments at CF centres included in the SPACE Study; and 4) all children had to be able to provide an exhaled breath sample into a collection bag.

\section{Sample size}

The power calculation was based on the expected rise in exhaled breath hydrogen cyanide concentration in those who acquired P. aeruginosa [17]. Aiming for a sensitivity and specificity of $95 \%$, it was estimated that exhaled breath hydrogen cyanide concentrations needed to be measured in 46 children at the time of $P$. aeruginosa infection. A retrospective audit at one of the recruiting centres found the rate of new $P$. aeruginosa infections to be 0.1 cases per patient-year. The total patient-years required to originate 46 new $P$. aeruginosa cases was therefore estimated to be 460 , which was achieved by following 230 patients for 2 years.

\section{Patient recruitment and follow-up}

A total of 233 children were recruited from eight paediatric CF centres across the Midlands and the North West of the UK. Their median (interquartile range (IQR)) age was 8.0 (5.0-12.2) years. At each centre, recruitment was open for 6 months but the study start date varied between centres for administrative reasons. The first child was recruited in June 2011 and the last child in October 2012. The study closed at all centres in December 2013. The median duration of follow-up was 2.0 (1.7-2.3) years. 10 children were withdrawn from the study prior to the close date: six transitioned to adult services and four moved to a different area. The data on these 10 children are included in the analysis up to the point at which they were withdrawn. 


\section{Collection and analysis of breath samples}

At each study visit, the children were invited to inflate a 1-L gas sampling bag made from $70-\mu m$-thick Nalophan using one long exhalation. Some younger children needed two or three exhalations. Once the bag was filled, the disposable mouthpiece was removed and the bag sealed. The samples were transported to Keele University (Keele, UK) for analysis using a Profile 3 SIFT-MS instrument (Trans Spectra Limited, Newcastle-under-Lyme, UK) [19, 20]. The bags were punctured with a hypodermic needle directly attached to the heated input arm of the SIFT-MS instrument. This enabled the exhaled breath gas to flow unhindered into the analytical region of the instrument. The mean concentrations of water vapour and hydrogen cyanide were analysed over $100 \mathrm{~s}$ while operating the instrument in the multiple ion monitoring mode [21]. Informed by previous studies, the bags/samples were heated to $37^{\circ} \mathrm{C}$ prior to analysis, which was carried out within $24 \mathrm{~h}$ of the sample being collected [22, 23]. In total, 2055 breath samples were analysed. On eight occasions, a child refused to provide a breath sample, and on 23 occasions, the breath sample was not analysed within $24 \mathrm{~h}$ due to problems with transport or with the SIFT-MS instrument.

\section{Study visits}

There was a total of 2086 study visits; the median (IQR) number of visits per patient-year was 4.8 (3.1-5.6). In addition to the breath sample, a clinical details questionnaire was completed by the child/ parent at each study visit. This collected data on the child's general state of health, and any change in cough, sputum production, shortness of breath or exercise tolerance. A respiratory microbiology sample was also taken at each study visit as part of the child's normal CF care. A sputum sample was taken from children who were able to expectorate and a cough swab was taken from those unable to expectorate. Induced sputum and BAL samples were not part of the study protocol, and no microbiology samples were taken at the request of the SPACE Study team. Despite this, if such samples were taken at the request of the clinical team, the results were included in the study analysis. To eliminate any bias by knowing from which children $P$. aeruginosa had been isolated, the SPACE Study team was only informed of any positive culture results at the end of the study.

\section{Statistical analysis}

The data are presented as median (IQR) values. The Mann-Whitney U-test was used to assess the difference between two groups and the Kruskal-Wallis test was used when there were more than two groups. The $P$. aeruginosa incidence rates (reported as cases per patient-year) were calculated as numerator/denominator. The numerator was the number of children with a new isolate of $P$. aeruginosa during the study period and the denominator was the "person-time at risk". The person-time at risk was the sum of the time from recruitment to the new P. aeruginosa isolate for those who isolated PA and the total follow-up time for those who remained P. aeruginosa infection free. The Poisson $95 \%$ confidence interval was calculated for the incidence rates and the Chi-squared test was used to compare the significance of two incidence rates. A p-value of $<0.05$ was deemed significant.

Given the repeated-measures nature of the data, a random-effects logistic model was used to account for the dependency between multiple observations from the same patient. As a guide to the estimated sensitivity and specificity, we dichotomised the predicted probabilities using a cut-off value of 0.5 and then estimated these quantities for clustered data [24]. The area under the receiver operator characteristics (ROC) curve was estimated using the clustered option in the SomersD option in STATA (StataCorp, College Station, TX, USA). To our knowledge, there is no standard method for calculating the Youden index for correlated data and it was therefore not possible to define the optimal cut-off hydrogen cyanide concentration that predicted $P$. aeruginosa infection.

\section{Ethical approval}

Ethical approval for the SPACE Study was granted by the Coventry and Warwickshire Research and Ethics Committee (ref. 10/H1211/48).

\section{Role of funding source}

The study sponsor had no role in the collection, analysis or interpretation of data; in the writing of the report; or in the decision to submit the paper for publication.

\section{Results}

\section{$P$, aeruginosa status}

None of the children recruited into the study had $P$. aeruginosa isolated from their sample for $\geqslant 12$ months. They were further separated into three groups according to their $P$. aeruginosa status: 113 children had never had $P$. aeruginosa grown from their samples (Never group), 99 had last $P$. aeruginosa isolated from their samples $>12$ months ago and were not receiving nebulised anti- $P$. aeruginosa therapy at 
study entry (Free From (FF) group), and 21 had last had $P$. aeruginosa isolated from their samples $>12$ months ago but had continued to receive prophylactic nebulised anti- $P$. aeruginosa therapy (Free From But Treated (FFT) group). All those in the FFT group had previously been diagnosed with chronic $P$. aeruginosa infection according to standard criteria [25]. The median age was lowest in the Never group and highest in the FFT group (table 1).

\section{Incidence of new P. aeruginosa isolates}

$P$. aeruginosa was isolated from 71 children during the study (29 in the Never group, 31 in the FF group and 11 in the FFT group). The median (IQR) recruitment ages were similar for those who subsequently grew $P$. aeruginosa and for those who remained $P$. aeruginosa infection free: 7.7 (4.4-12.4) versus 8.2 (5.1-12.1) years, respectively $(\mathrm{p}=0.71)$. The overall incidence $(95 \% \mathrm{CI})$ of new $P$. aeruginosa isolates was $0.19(0.15-0.23)$ cases per patient-year. The incidence rate $(95 \% \mathrm{CI})$ was higher in the FFT group than in the Never and FF groups combined: $0.41(0.20-0.73)$ versus $0.17(0.14-0.22)$ cases per patient-year, respectively (Chi-squared 7.41, p=0.007) (table 1). The percentage of patients remaining P. aeruginosa infection free at the study end was $74 \%$ in the Never group, $69 \%$ in the FF group and $48 \%$ in the FFT group (figure 1).

\section{Recruiting centres}

Table 2 shows the study activity and $P$. aeruginosa incidence rates in the eight centres. As we were comparing the incidence rate of new $P$. aeruginosa infection between centres, we excluded children in the FFT group as they had all previously been diagnosed with chronic $P$. aeruginosa infection and may only have been culture negative due to nebulised anti- $P$. aeruginosa prophylaxis. The proportion of children remaining $P$. aeruginosa free at the study end varied from $56 \%$ (centre 3 ) to $83 \%$ (centre 7 ). Centre 4 had less frequent routine outpatient appointments and, therefore, a lower number of study visits per patient-year.

\section{Pairing of study visits and P. aeruginosa culture results}

57 of the 71 children from whom $P$. aeruginosa was isolated had a study visit (with completed clinical details questionnaire and an analysed breath sample) on the day of the positive $P$. aeruginosa culture. In the remaining 14 children, the sample from which $P$. aeruginosa was isolated was not taken at a study visit; eight were postal samples, four were taken at an unscheduled hospital visit and two took place at visits of which the study team were unaware. In these 14 children, the gap between the positive culture and the next study visit was 1-5 weeks, and so the breath analysis and symptom questionnaire data were not used in the subsequent analysis.

\section{Symptoms at the time of $\mathrm{P}$. aeruginosa isolation}

33 (58\%) out of 57 children were symptomatic at the time of $P$. aeruginosa isolation. Of these, 30 (91\%) had increased cough, $16(48 \%)$ had increased sputum volume, nine $(27 \%)$ had increased shortness of breath and seven (21\%) had reduced exercise tolerance. Only 12 (36\%) symptomatic children were described as "unwell" or "less well than normal".

\section{TABLE 1 Pseudomonas aeruginosa incidence rates, study activity and age according to}

$P$. aeruginosa status

\begin{tabular}{|c|c|c|c|c|c|}
\hline \multirow{2}{*}{$\begin{array}{l}P . \text { aeruginosa } \\
\text { status }\end{array}$} & \multirow[t]{2}{*}{ Recruits } & \multirow{2}{*}{$\begin{array}{c}\text { Age years } \\
\text { median (IQR) }\end{array}$} & \multicolumn{2}{|c|}{ Study activity } & \multirow{2}{*}{$\begin{array}{c}P \text {. aeruginosa incidence } \\
\text { cases per patient-year } \\
(95 \% \mathrm{CI})\end{array}$} \\
\hline & & & $\begin{array}{l}\text { Total } \\
\text { visits }\end{array}$ & $\begin{array}{l}\text { Visits per } \\
\text { patient-year } \\
\text { median (IQR) }\end{array}$ & \\
\hline Never & 113 & $6.4(4.0-10.9)$ & 962 & $4.5(3.1-5.4)$ & $0.15(0.10-0.22)$ \\
\hline FF & 99 & $8.1(5.3-12.5)$ & 910 & $5.0(3.7-5.6)$ & $0.19(0.13-0.27)$ \\
\hline FFT & 21 & $13.1(10.6-15.5)$ & 214 & $5.9(3.2-6.5)$ & $0.41(0.20-0.73)$ \\
\hline Total & 233 & $8.0(5.0-12.2)$ & 2086 & $4.8(3.1-5.6)$ & $0.19(0.15-0.23)$ \\
\hline
\end{tabular}


FIGURE 1 Kaplan-Meier curve showing the proportion of children remaining free from Pseudomonas aeruginosa infection during the study period for each $P$. aeruginosa status group. FF: Free From; FFT: Free From But Treated.

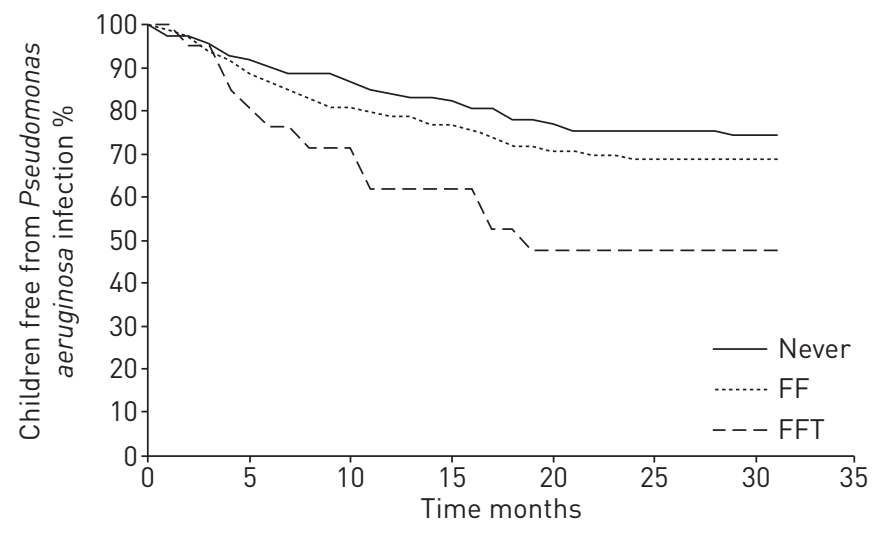

\section{Breath hydrogen cyanide concentrations}

At the first study visit, there was no difference in the median (IQR) exhaled breath hydrogen cyanide concentrations in parts per billion by volume (ppbv) between the three P. aeruginosa status groups: Never, 2.2 (1.3-3.2); FF, 2.3 (1.5-3.0); and FFT 2.4 (1.2-3.5) ppbv ( $\mathrm{p}=0.58)$. The 162 children who remained $P$. aeruginosa free throughout the study provided 1398 breath samples (figure 2). The median hydrogen cyanide concentration was $2.3(1.3-3.0) \mathrm{ppbv}$. The median (IQR) difference between an individual's minimum and maximum hydrogen cyanide concentration (intrasubject variation) was 2.8 (1.9-3.5) ppbv. The hydrogen cyanide concentration on the day of $P$. aeruginosa isolation is shown in figure 3 for the 57 children included in this analysis. The first peak of this bimodal distribution contains breath samples from 29 patients and has a median hydrogen cyanide concentration of 2.2 ppbv. This has a similar distribution to that seen in the $P$. aeruginosa negative patients in figure 2 . The second peak contains breath samples from 28 patients and has a median hydrogen cyanide concentration of 6.3 ppbv. All the breath samples in this second peak had a hydrogen cyanide concentration $>5.0$ ppbv. There was no significant difference between the children in the first and second peaks in terms of age or P. aeruginosa status. Five of the 28 children in the second peak had a hydrogen cyanide concentration $\geqslant 5.0 \mathrm{ppbv}$ for up to two visits prior to the date of the positive $P$. aeruginosa culture and the hydrogen cyanide remained $>5.0 \mathrm{ppbv}$ in eight children for up to two visits after the positive P. aeruginosa culture date.

\section{Modelling longitudinal hydrogen cyanide concentration}

A random-effects logistic model was used to account for the dependency between multiple observations from the same patient. The estimated odds ratio (95\% CI) was $3.12(2.58-3.56)$, which showed that for a 1 -ppbv increase in exhaled breath hydrogen cyanide, we expected a $212 \%$ increase in the odds of $P$. aeruginosa infection. The estimated sensitivity (95\% CI) was 33\% (22-44\%) and the estimated

\section{TABLE 2 Pseudomonas aeruginosa incidence rates, study activity and age according to} recruiting centre

\begin{tabular}{lccccc} 
Centre & Recruits & $\begin{array}{c}\text { Age years } \\
\text { median (IQR) }\end{array}$ & \multicolumn{2}{c}{ Study activity } & $\begin{array}{c}\text { P. aeruginosa incidence cases } \\
\text { per patient-year (95\% CI) }\end{array}$ \\
\cline { 4 - 5 } & & & $\begin{array}{c}\text { Total } \\
\text { visits }\end{array}$ & $\begin{array}{c}\text { Visits per patient } \\
\text { year median (IQR) }\end{array}$ & \\
\hline $\mathbf{1}$ & 24 & $7.6(4.0-8.0)$ & 188 & $5.7(5.3-6.3)$ & $0.22(0.10-0.40)$ \\
$\mathbf{2}$ & 34 & $8.8(6.0-12.0)$ & 348 & $4.9(4.3-5.2)$ & $0.18(0.10-0.29)$ \\
$\mathbf{3}$ & 21 & $6.9(4.9-13.2)$ & 193 & $4.2(3.5-4.5)$ & $0.27(0.15-0.43)$ \\
$\mathbf{4}$ & 53 & $9.1(5.4-12.5)$ & 229 & $2.4(1.9-2.8)$ & $0.20(0.12-0.29)$ \\
$\mathbf{5}$ & 30 & $9.4(6.7-12.9)$ & 306 & $5.2(4.5-5.5)$ & $0.13(0.07-0.25)$ \\
$\mathbf{6}$ & 18 & $8.0(2.4-10.7)$ & 136 & $4.5(3.7-5.3)$ & $0.28(0.14-0.49)$ \\
$\mathbf{7}$ & 36 & $5.2(3.3-8.5)$ & 477 & $5.9(5.2-6.7)$ & $0.08(0.04-0.18)$ \\
$\mathbf{8}$ & 17 & $8.4(5.2-12.0)$ & 209 & $5.5(4.9-6.2)$ & $0.22(0.10-0.40)$ \\
Total & 233 & $8.0(5.0-12.2)$ & 2086 & $4.8(3.1-5.6)$ & $0.17(0.14-0.22)$ \\
\hline
\end{tabular}

Data are presented as $\mathrm{n}$ unless otherwise stated. Children in the Free From But Treated group were excluded when the $P$. aeruginosa incidence and antibiotic courses were calculated. IQR: interquartile range. 

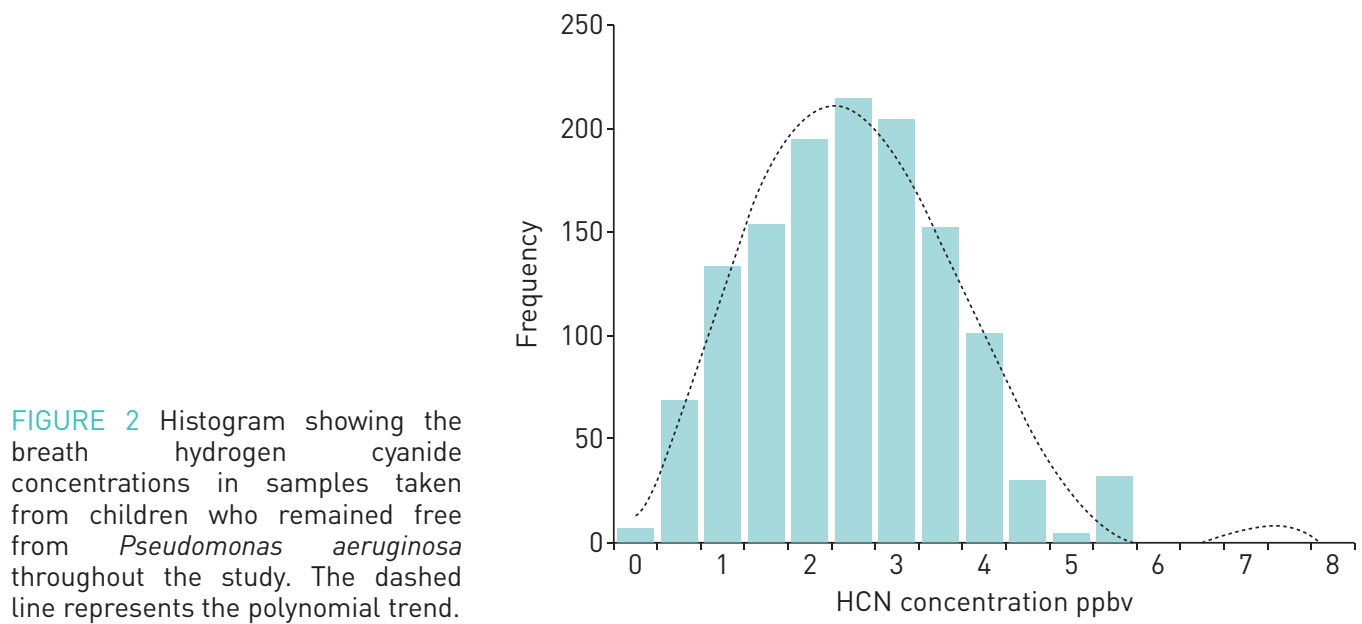

specificity (95\% CI) was 99\% (99.2-99.9\%). The area under the ROC curve was estimated as 0.88 (0.83-0.94). This equates to the probability that a child with a new $P$. aeruginosa infection will have higher breath hydrogen cyanide than one who remains free from $P$. aeruginosa.

\section{Discussion}

The SPACE Study is one of the largest breath analysis studies to be undertaken and is the first to investigate whether exhaled breath hydrogen cyanide can be used as an early biomarker of P. aeruginosa infection in children with CF. As well as demonstrating that hydrogen cyanide is a specific biomarker of $P$. aeruginosa infection, it has provided novel prospective data on the incidence of new $P$. aeruginosa infection in children previously free from $P$. aeruginosa infection.

The high specificity with which elevated exhaled breath hydrogen cyanide indicates early P. aeruginosa infection is very encouraging and suggests that it may have a role in the identification of this infection. It must also be recognised that this study did not routinely use BAL culture, which is the gold standard method of $P$. aeruginosa detection in nonexpectorating patients. We therefore relied on cough swabs, which only have a sensitivity of $44-82 \%[6,7]$. It is of particular interest that five children had breath hydrogen cyanide $>5.0 \mathrm{ppbv}$ for one or two study visits prior to the date of their positive P. aeruginosa culture. Although this is only a small number of children, it shows that in certain situations, exhaled breath hydrogen cyanide may indicate the presence of $P$. aeruginosa infection that has been missed on routine microbiology samples.

The low sensitivity with which elevated exhaled breath hydrogen cyanide predicted $P$. aeruginosa infection was ultimately disappointing. The hydrogen cyanide concentrations at the time of $P$. aeruginosa isolation showed a bimodal distribution (figure 3). The distribution of the first peak was very similar to that seen in the children who remained free from $P$. aeruginosa throughout the study (figure 2). This suggests that in approximately half of the children with a new $P$. aeruginosa isolate, there was little effect on their exhaled

FIGURE 3 Histogram showing the bimodal distribution of breath hydrogen cyanide concentrations taken from children at the time of Pseudomonas aeruginosa isolation. The dashed line represents the polynomial trend.

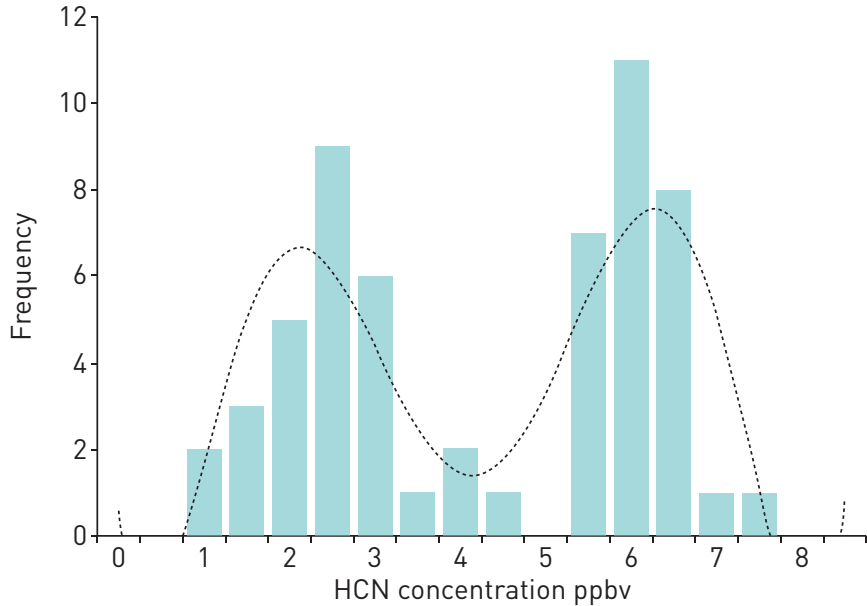


breath hydrogen cyanide. There are a number of possible explanations for this. One factor is the genotype of the infecting $P$. aeruginosa. Our in vitro studies have shown that $P$. aeruginosa releases hydrogen cyanide into the gas phase, which is detectable in the culture headspace [12]. When multiple $P$. aeruginosa genotypes were analysed, they all produced hydrogen cyanide but there was a $>700$-fold difference in the maximum hydrogen cyanide concentration produced by the various strains [13]. Although the exact correlation between the in vitro headspace concentrations and the in vivo exhaled breath hydrogen cyanide concentrations is contentious, it is reasonable to presume that pulmonary infection with one of the low hydrogen cyanide producing strains may not produce enough hydrogen cyanide to be detected in exhaled breath.

Another factor that may lead to low sensitivity is the methodology of the hydrogen cyanide analysis. The SPACE Study involved the analysis of breath samples taken at multiple CF centres spread over a wide geographic area. Therefore, the only practical solution was to collect the breath samples in bags and transport them to the SIFT-MS instrument at Keele University for analysis. When bag samples are pre-warmed to $37^{\circ} \mathrm{C}$ and analysis occurs within $24 \mathrm{~h}$ of collection, there is good correlation between these "offline" hydrogen cyanide concentrations and the "online" results obtained by the individual breathing directly into the SIFT-MS instrument [22]. Despite the good correlation, offline concentrations are lower, which increases the chance of false-negative measurements. The technology to allow accurate hydrogen cyanide analysis at even lower concentrations, and the use of smaller and more portable devices continues to improve. This raises the possibility of being able to take regular online measurements in the outpatient clinic.

As with all breath trace gas metabolites, there is intrasubject variation in exhaled breath hydrogen cyanide concentrations [17]. Factors that contribute to this include diet and the time of day the sample is taken [26]. Although the children in the SPACE study were requested not to eat or drink for $30 \mathrm{~min}$ prior to providing an exhaled breath sample, it was not possible to accurately confirm this and due to the different timings of the CF clinics, it was not possible to standardise the sample time. Other factors that may have affected the sensitivity are the use of mouth-exhaled rather than nose-exhaled breath samples and the effect of younger children using multiple exhalations to fill the bag $[18,27]$.

Although the UK CF Trust Patient Registry provides data on the prevalence of chronic P. aeruginosa infection, it does not provide data on the incidence of new P. aeruginosa infection. We believe that the SPACE Study is the first to provide this data. Interesting differences were seen between the $P$. aeruginosa status groups and the different centres. The higher incidence of new $P$. aeruginosa infection in the FFT group compared to the Never and FF groups is not surprising since these children had all been started on long-term nebulised anti- $P$. aeruginosa treatment after being diagnosed with chronic $P$. aeruginosa infection. It is generally assumed that once chronic $P$. aeruginosa infection is confirmed, the organism will continue to be isolated from the patient [28]. The results in the FFT group demonstrate that when such patients are commenced on nebulised anti- $P$. aeruginosa treatment, suppression of the infection may result in $P$. aeruginosa not being isolated for long periods. Therefore, these patients no longer meet the criteria for chronic P. aeruginosa infection. How they should be classified on national databases is also open to debate. We were interested in further investigating the variation in $P$. aeruginosa incidence rates between the centres. The exact infection control practice at each centre was clarified but no single practice could be found to explain the difference in $P$. aeruginosa incidence rates. Other factors that may have influenced the incidence rates include the number of patients at each centre, the age of the patients, the model of care at each centre, the severity of the children's lung disease, and the social interaction and behaviour of the patients and their families.

\section{Summary}

This study has demonstrated that exhaled hydrogen cyanide is a specific biomarker of new P. aeruginosa infection in children with CF. Its low sensitivity means that at present, hydrogen cyanide cannot be used as a screening test for early $P$. aeruginosa infection. With continued advances in the sensitivity of SIFT-MS technology, and the development of smaller and more portable instruments, this may change in the future. The SPACE Study has also provided novel prospective data on the incidence of new P. aeruginosa infection, which may prompt further research on infection control measures and help inform the design of future trials, particularly in relation to $P$. aeruginosa eradication.

\section{Acknowledgments}

The SPACE Study Team collaborators are G. Aminson (Birmingham Children Hospital, Birmingham, UK), C. Beane (Manchester Children Hospital, Manchester, UK), S.A. Bell (Royal Derby Hospital, Derby, UK), J. Bradley (Birmingham Children Hospital, Birmingham, UK), M. Callaghan (Queens Medical Centre, Nottingham, UK), K. Castro-Foskett (Royal Stoke University Hospital, Stoke on Trent, UK), T. Chippendale (Royal Stoke University Hospital, Stoke on Trent, UK), J. Clark (Alder Hey Children's Hospital, Liverpool, UK), K. Cooke (Royal Stoke University Hospital, Stoke on Trent, UK), C. Cotter (Birmingham Children Hospital, Birmingham, UK), L. Crate (Queens Medical Centre, Nottingham, UK), L. de Bourg (Royal Stoke University Hospital, Stoke on Trent, UK), M. Desai (Birmingham Children Hospital, Birmingham, UK), L. Fear (Queens Medical Centre, Nottingham, UK), C. Fish (Manchester Children Hospital, Manchester, UK), A. Frost (Queens Medical Centre, Nottingham, UK), D.A. Harrison (Royal Stoke University Hospital, 
Stoke on Trent, UK), F. Hay (Queens Medical Centre, Nottingham, UK), R. Jones (Royal Stoke University Hospital, Stoke on Trent, UK), S. Kempsey (Royal Wolverhampton Hospital, Wolverhampton, UK), M. MacGregor (Royal Derby Hospital, Derby, UK), P. McCormack (Alder Hey Children's Hospital, Liverpool, UK), C. Miller (Shrewsbury and Telford Hospitals NHS Trust, Telford, UK), C. Owen (Shrewsbury and Telford Hospitals NHS Trust, Telford, UK),

J. Panickar (Manchester Children Hospital, Manchester, UK), H. Parker (Royal Stoke University Hospital, Stoke on Trent, UK), M. Phipps (Royal Stoke University Hospital, Stoke on Trent, UK), R. Raynor (Royal Wolverhampton Hospital, Wolverhampton, UK), M. Rees (Shrewsbury and Telford Hospitals NHS Trust, Telford, UK), E. Roe (Royal Stoke University Hospital, Stoke on Trent, UK), N. Ruggins (Royal Derby Hospital, Derby, UK), C. Smith (Royal Derby Hospital, Derby, UK), J. Tomlinson (Royal Stoke University Hospital, Stoke on Trent, UK), V. Unsworth (Royal Derby Hospital, Derby, UK) and N. Watson (Royal Derby Hospital, Derby, UK).

We would like to thank the principal investigators, research nurses and coordinators at each of the recruiting centres for their hard work and dedication. We would also like to thank all the patients and families that supported the study.

\section{References}

1 Emerson J, Rosenfeld M, McNamara S, et al. Pseudomonas aeruginosa and other predictors of mortality and morbidity in young children with cystic fibrosis. Pediatr Pulmonol 2002; 34: 91-100.

2 Nixon GM, Armstrong DS, Carzino R, et al. Clinical outcome after early Pseudomonas aeruginosa infection in cystic fibrosis. J Pediatr 2001; 138: 699-704.

3 Valerius NH, Koch C, Høiby N. Prevention of chronic Pseudomonas aeruginosa colonisation in cystic fibrosis by early treatment. Lancet 1991; 338: 725-726.

4 Langton Hewer SC, Smyth AR. Antibiotic strategies for eradicating Pseudomonas aeruginosa in people with cystic fibrosis. Cochrane Database Syst Rev 2009: CD004197.

5 Douglas TA, Brennan S, Gard S, et al. Acquisition and eradication of $P$. aeruginosa in young children with cystic fibrosis. Eur Respir J 2009; 33: 305-311.

6 Rosenfeld M, Emerson J, Accurso F, et al. Diagnostic accuracy of oropharyngeal cultures in infants and young children with cystic fibrosis. Pediatr Pulmonol 1999; 28: 321-328.

7 Armstrong DS, Grimwood K, Carlin JB, et al. Bronchoalveolar lavage or oropharyngeal cultures to identify lower respiratory pathogens in infants with cystic fibrosis. Pediatr Pulmonol 1996; 21: 267-275.

8 De Boeck K, Alifier M, Vandeputte S. Sputum induction in young cystic fibrosis patients. Eur Respir J 2000; 16: 91-94.

9 Al-Saleh S, Dell SD, Grasemann H, et al. Sputum induction in routine clinical care of children with cystic fibrosis. J Pediatr 2010; 157: 1006-1011.e1.

10 Brennan S, Gangell C, Wainwright C, et al. Disease surveillance using bronchoalveolar lavage. Paediatr Respir Rev 2008; 9: 151-159.

11 Clawson BJ, Young CC. Preliminary report on the production of hydrocyanic acid by bacteria. J Biol Chem 1913, 15: 419-422.

12 Carroll W, Lenney W, Wang T, et al. Detection of volatile compounds emitted by Pseudomonas aeruginosa using selected ion flow tube mass spectrometry. Pediatr Pulmonol 2005; 39: 452-456.

13 Gilchrist FJ, Alcock A, Belcher J, et al. Variation in hydrogen cyanide production between different strains of Pseudomonas aeruginosa. Eur Respir J 2011; 38: 409-414.

14 Gilchrist FJ, Sims H, Alcock A, et al. Quantification of hydrogen cyanide and 2-aminoacetophenone in the headspace of Pseudomonas aeruginosa cultured under biofilm and planktonic conditions. Anal Methods 2012; 4 3661-3665.

15 Williams HD, Zlosnik JEA, Ryall B. Oxygen, cyanide and energy generation in the cystic fibrosis pathogen Pseudomonas aeruginosa. Adv Microb Physiol 2007; 52: 1-71

16 Gilchrist FJ, Sims H, Alcock A, et al. Is hydrogen cyanide a marker of Burkholderia cepacia complex? J Clin Microbiol 2013; 51: 3849-3851.

17 Enderby B, Smith D, Carroll W, et al. Hydrogen cyanide as a biomarker for Pseudomonas aeruginosa in the breath of children with cystic fibrosis. Pediatr Pulmonol 2009; 44: 142-147.

18 Gilchrist FJ, Bright-Thomas RJ, Jones AM, et al. Hydrogen cyanide concentrations in the breath of adult cystic fibrosis patients with and without Pseudomonas aeruginosa infection. J Breath Res 2013; 7: 026010.

19 Spaněl P, Smith D. Progress in SIFT-MS: breath analysis and other applications. Mass Spectrom Rev 2011; 30 236-267.

20 Spanel P, Dryahina K, Smith D. A general method for the calculation of absolute trace gas concentrations in air and breath from selected ion flow tube mass spectrometry data. Int J Mass Spectrom 2006; 249: 230-239.

21 Španěl PSD. Selected Ion Flow Tube mass Spectrometry (SIFT-MS) for on-line trace gas analysis of breath. Clin Diagn Ther Monit. 2005; 3-34.

22 Gilchrist FJ, Razavi C, Webb AK, et al. An investigation of suitable bag materials for the collection and storage of breath samples containing hydrogen cyanide. J Breath Res 2012; 6: 036004.

23 Spaněl P, Wang T, Smith D. Quantification of hydrogen cyanide in humid air by selected ion flow tube mass spectrometry. Rapid Commun Mass Spectrom 2004; 18: 1869-1873.

24 Genders TSS, Spronk S, Stijnen T, et al. Methods for calculating sensitivity and specificity of clustered data: a tutorial. Radiology 2012; 265: 910-916.

25 Lee TWR, Brownlee KG, Conway SP, et al. Evaluation of a new definition for chronic Pseudomonas aeruginosa infection in cystic fibrosis patients. J Cyst Fibros 2003; 2: 29-34.

26 Schmidt FM, Metsälä M, Vaittinen O, et al. Background levels and diurnal variations of hydrogen cyanide in breath and emitted from skin. I Breath Res 2011; 5: 046004.

27 Wang T, Pysanenko A, Dryahina K, et al. Analysis of breath, exhaled via the mouth and nose, and the air in the oral cavity. J Breath Res 2008; 2: 037013.

28 Lee TWR. Eradication of early Pseudomonas infection in cystic fibrosis. Chron Respir Dis 2009; 6: 99-107. 